\title{
Free light chains in the cerebrospinal fluid: an indicator of recent immunological stimulation
}

\author{
A VAKAET, ${ }^{*}$ EJ THOMPSON $\dagger$ \\ From the Departments of Clinical Neurophysiology* and Clinical Neurochemistry $\dagger$ The National Hospital, \\ London, UK
}

SUMMARY A group of 38 patients with immunoglobulin free light chains (kappa and/or lambda) in the cerebrospinal fluid was studied. Only five of them showed one band of kappa free light chains, 36 of them showed one or more bands of lambda free light chains. There were 33 multiple sclerosis patients among them and they were compared with 33 control multiple sclerosis patients who did not have free light chains but only showed an oligoclonal pattern of immunoglobulins in their CSF. Of several clinical and pathological parameters, only three were statistically significant: (A) for all patients, as well as for the multiple sclerosis patients alone, elevated numbers of free light chains of type lambda were associated with the elevated numbers of white blood cells in the CSF ( $p<0.003$ ), (B) for multiple sclerosis patients, elevated numbers of free light chains of type lambda were associated with shorter time intervals between the last relapse and the date of the lumbar puncture $(p<0.004)$ and $(C)$ also with shorter duration of the disease $(p<0.01)$. The presence of lambda free light chains in the cerebrospinal fluid seems to indicate that recent antigenic stimulation has occurred within the central nervous system following exacerbation. The inverse association of the number of lambda free light chains with the duration of the disease is suggestive of a pathological expression of the "burning out" phenomenon of the multiple sclerosis process.

Immunological theories have opened new horizons to our knowledge of several neurological diseases (for example multiple sclerosis, Guillain-Barré syndrome, and myasthenia gravis). Studies of parameters of the general immunological system as well as of the immunological system within the confines of the central nervous system (CNS) itself, have given clues about possible aetiological mechanisms. $^{12}$

The immunoglobulins, of which IgG is the major representative are the best known immunological parameters of CNS immune reactions. They can be detected in normal cerebrospinal fluid but their concentration in the CSF is closely related to their concentration in serum as well as the state of the blood-CSF-barriers. Changes of these ratios are associated with various pathological conditions. ${ }^{34}$

\footnotetext{
Address for reprint requests: Dr EJ Thompson, Department of Clinical Neurochemistry, The National Hospital for Nervous Diseases, Queen Square, London WC1N 3BG, UK.
}

Received 25 September 1984 and in revised form 5 February 1985. Accepted 9 February 1985
Each IgG molecule contains two identical heavy chains (type gamma) and two identical light chains (either type kappa or type lambda). In the general IgG population, there are roughly equal proportions of individual molecules, each containing either kappa or lambda light chains. The presence of free light chains, that is, not bound to any heavy chains, is well known to occur in the urine and/or CSF of patients with myeloma, in which case they are called Bence-Jones protein (being a monolithic product of the tumour cells). The present study aims to throw some light on the meaning of the presence of one or more free light chains in the CSF of patients with other pathological conditions.

\section{Materials and methods}

A method to detect free light chains in the CSF has been elaborated, using the replicate immunoblotting technique. ${ }^{2}$ Briefly, the CSF proteins were separated by polyacrylamide gel electrophoresis and the free light chains, that is those outside the gamma region, were counted using 


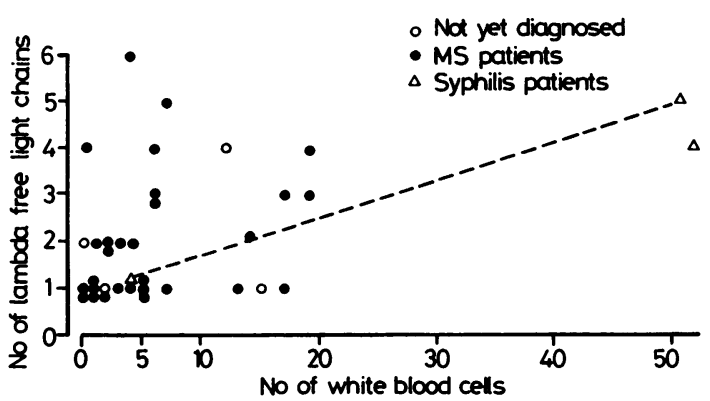

Fig 1 Plot of numbers of lambda free light chains (ordinate) versus the numbers of CSF white blood cells per $\mu l$ (abscissa). The broken line connects two specimens from the same patient with neurosyphilis at times before (right) and after (left) penicillin therapy. Filled circles represent multiple sclerosis patient samples, open circles represent those from patients not yet diagnosed and crosses those from patients with neurosyphilis. Taking the entire group, when the numbers of patients with pleocytosis $(6$ or more white cells $\mu l)$ were compared with patients having 3 or more free light chains (FLC) in their CSF, $(n=36)$ the Fischer exact test gave a probability for difference of $p<0.002$. Taking the subgroup of those with multiple sclerosis, when the numbers of patients with pleocytosis (6 or more white cells per $\mu l)$ were compared with patients having 3 or more free light chains $(F L C)$ in their CSF, $(n=30)$ the Fischer exact test gave a probability for difference of $p<0.003$.

previously described techniques. ${ }^{5}$ We have found that by immunofixation for IgG, the intact molecule is restricted to the gamma 2 to 5 region, and $\operatorname{IgA}$ is in the gamma 1 to 3 region, while IgM is found post alpha 2 macroglobulin. Parallel immunofixation of replicate nitrocellulose blots has shown that kappa and/or lambda light chains occur in regions more anodic than gamma 1 to 5 and since there are no corresponding heavy chains $(G, A$ or $M)$ they clearly represent free light chains.

This technique has been applied routinely for about a year on all CSF specimens which were received by the laboratory. From some 3,500 samples, we selected about 100 sequential (random) patients whose CSF showed the presence of free light chains. We then retrospectively managed to gather reliable clinical data on 38 patients from studies of the case notes and/or discharge summaries. From the same time period, we also selected 33 sequential (ran-

Table 1 List of diagnoses for patients with and without free light chains (FLC) of type lambda and kappa

\begin{tabular}{|c|c|c|}
\hline & With FLC & Without FLC \\
\hline $\begin{array}{l}\text { Multiple sclerosis (total) } \\
\text { clinically definite } \\
\text { early probable } \\
\text { progressive probable } \\
\text { progressive possible } \\
\text { suspected }\end{array}$ & $\begin{array}{rr}33 & \\
17 \\
9 \\
2 \\
\\
0 \\
5\end{array}$ & $\begin{array}{rr}33 & \\
22 \\
2 \\
4 \\
2 \\
3\end{array}$ \\
\hline $\begin{array}{l}\text { Neurosyphilis } \\
\text { Not yet diagnosed } \\
\text { Totals }\end{array}$ & $\begin{array}{r}2 \\
3 \\
38\end{array}$ & $\begin{array}{r}\mathbf{0} \\
\mathbf{0} \\
\mathbf{3 3}\end{array}$ \\
\hline
\end{tabular}

Table 2 Qualitative (QUAL) and quantitative (QUAN) comparison of patients with and without free light chains of type lambda for various clinical parameters. Not significant (NS) or significant (S) associations were found

Age

Sex

Age at onset of disease

Duration of disease

Total number of attacks

Number of attacks in year preceding sample date

Number of attacks divided by duration

Grade of disability (Kurtzke) - general status - separate functional systems

Grade of general disability divided by duration

Time interval between beginning of last relapse and sample date

(that is lumbar puncture date)

$\begin{array}{ll}\text { QUAL } & \text { QUAN } \\ \text { NS } & \text { NS } \\ \text { NS } & \text { NS } \\ \text { NS } & \text { NS } \\ \text { NS } & \text { S } \\ \text { NS } & \text { NS } \\ \text { NS } & \text { NS } \\ \text { NS } & \text { NS } \\ \text { NS } & \text { NS } \\ \text { NS } & \text { NS } \\ \text { NS } & \text { NS } \\ & \\ \text { NS } & \text { S }\end{array}$

dom) multiple sclerosis patients who did not have free light chains. Patients with multiple myeloma or related diseases (paraproteins) were excluded.

The clinical and immunochemical data was assessed blind and prior to performing the Fischer exact test. Although one appreciates the limitations of retrospective studies of hospital notes, nevertheless any difficulties would apply equally to all cases and therefore would not influence the validity of the statistical conclusions.

\section{Results}

We studied 39 CSF samples from the 38 patients selected. All these patients showed an oligoclonal pattern of immunoglobulins. There were 34 samples which contained only lambda free light chains (from 1 to 6), three samples contained only one kappa and two samples showed concurrence of one lambda and one kappa free light chains. Because of the small number of patients with kappa free light chains, no

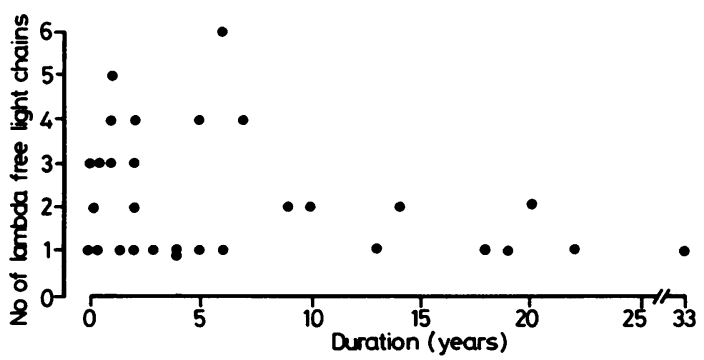

Fig 2 Plot of numbers of lambda free light chains (ordinate) versus the duration of multiple sclerosis in years (abscissa). When the numbers of patients with 3 or more lambda free light chains (FLC) were compared with duration of multiple sclerosis for up to 7 years or longer, the Fischer exact test $(n=30)$ showed a probability of $p<0.01$. 


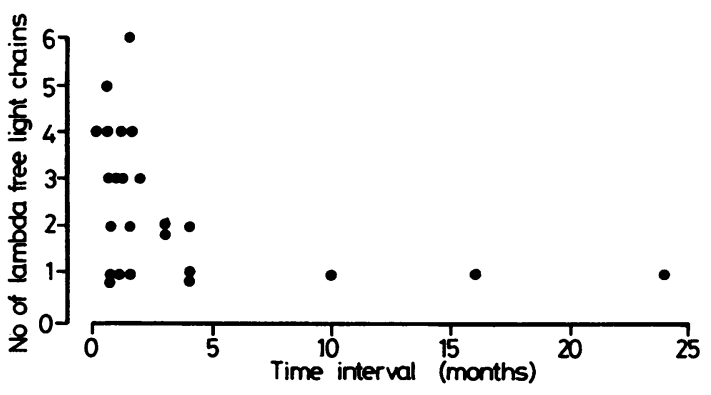

Fig 3 Plot of numbers of lambda free light chains (ordinate) versus the time interval from last relapse to the date of lumbar fuid sample in months (abscissa) in patients with multiple sclerosis. When the numbers of patients with 3 or more lambda free light chains (FLC) were compared with interval to last relapse of up to 2 months, the Fischer exact test $(n=24)$, showed a probability of $<0.004$.

conclusions can be drawn as to the meaning of their occurrence. We therefore focused our attention solely on the patients with lambda free light chains. The patients who showed both one kappa and one lambda light chain were accepted as having one lambda free light chain. As for pathological parameters, there was no statistically significant correlation between the number of free light chains and the total CSF protein. However, there was an association between pleocytosis and elevated numbers of free light chains (fig. 1). The white blood cell count of the CSF has been corrected if red blood cells were also shown to be present, according to the peripheral blood count. The association shown was statistically significant, either when considering all disease processes $(p<0.002)$ or when only multiple sclerosis patients were taken into account $(p<$ 0.003 ). The list of diagnoses of the 38 patients studied is shown in table 1 . The diagnosis of multiple sclerosis has been further subclassified according to the criteria of McDonald and Halliday. ${ }^{6}$ The two neurosyphilis patients had the highest white blood cell counts also had a large number of free light chains in the CSF. In one case, we were able to study two samples of CSF, before and after penicillin treatment. The first sample showed 51 white blood cells and five lambda chains, while the second showed only four white cells and one lambda chain.

As for the clinical data, we have focused mainly on the multiple sclerosis patients. Of these patients, 30 showed free light chains of type lambda, two of them additionally showed one free light chain type kappa, and three patients showed one free light chain type kappa. We paired them with a control group of 33 multiple sclerosis patients, who all showed an oligoclonal pattern of immunoglobulins (as all the multiple sclerosis patients with free light chains did) but they lacked free light chains. The distribution of the subtypes of multiple sclerosis patients is shown in table 1 . We have compared both groups for the clinical parameters mentioned in table 2 to see if the two groups (with and without free light chains) differed qualitatively. We also looked for a quantitative association of these parameters with the number of free light chains type lambda, and the results of which are shown in table 2 . The grade of disability has been determined using the Kurtzke scale, for the general condition and for each functional system separately. ${ }^{7}$ Two associations were statistically significant. (1) The number of free lambda light chain bands was inversely related to the duration of the disease $(p<0.01)($ fig. 2$)$. We found that when the disease process had lasted for a period of more than 7 years, no patient showed more than two lambda free light chains in the CSF. This association with duration of disease was not biased by the type of multiple sclerosis, (classified according to the McDonald and Halliday criteria) or by the Kurtzke grade of disability. (2) The inverse association of the number of lambda free light chains with the time interval between the last relapse and the date of the lumbar puncture was also significant $(p<0.004)$, although this information was available for only 24 of the multiple sclerosis patients (fig. 3). It can be seen that in all patients with three or more free light chains, the relapse had occurred within the last 2 months (from sample date). We have taken the definition of a relapse, as it is stated by Schumacher et al: a period of at least 24 hours in which there is worsening of (an) existing symptom(s) provided that the course has been stationary or has improved during the previous month. ${ }^{8}$

\section{Discussion}

The technique of immunoblotting polyacrylamide gels will detect free light chains which are not bound to heavy chain immunoglobulins. In our 38 patients studied, 33 showed up to six lambda bands and only five showed a single kappa band of free light chains. No conclusions about the presence of kappa chains can be drawn owing to the small number of such patients. However the difference between the absolute number of patients with kappa and with lambda free light chains is noteworthy in itself. Using a technique which is less direct than electrophoresis, in 1975, Bollengier et al suggested that free light chains occurred in CSF. ${ }^{9}$ However, it is worth noting that, using their rather different technique of immunoprecipitation with antiserum directed against Bence-Jones protein which had been previously adsorbed with heavy chains, the number of 
patients with free kappa was found to be larger than those with free lambda. Bollengier, in 1979 discussed some of the difficulties of comparing her data with others as well as her previous data. ${ }^{10}$

The other technique which is rather more akin to our own is that of electrophoresis followed by immunodiffusion." "Using this approach, it has been found that $96 \%$ of multiple sclerosis patients had elongation of their kappa and/or lambda precipitin arcs. It is not possible to count the number of individual bands of light chains with this particular technique; however, they comment that most of the elongations were anodic. This may imply agreement with our own findings, but it should also be noted that being near the origin in their system (due to electroendosmosis) they may also be detecting immune complexes, which are retarded near to the cathode in acrylamide electrophoresis due to molecular seiving by the gel.

Elevated numbers of free light chains were associated with elevated numbers of CSF white cells (pleocytosis). Moreover, the larger number of free light chains is also related to relatively recent clinical exacerbations. In our laboratory, the presence of three or more free light chains (always type lambda) points to a relapse having occurred within 2 months of the date of the lumbar sample. It is known that in other circumstances such as bone marrow transplantation and animals raised in germ-free environments, that the initial encounter with antigens is associated with the presence of free light chains. Taking into account our own findings, the presence of free light chains in the CSF could well be an indicator that recent immunological stimulation has taken place within the CNS.

The second finding is the inverse relationship between the number of free lambda light chains and the duration of the disease. The question as to whether this is a pathological expression of the "burning out" phenomenon of the multiple sclerosis process deserves further study.

There is an additional problem which also requires further investigation: why do only a proportion of multiple sclerosis patients show free light chains in the CSF and yet this group does not seem to differ clinically from the control group? It has not been possible to discern a difference from our retrospective study of hospital notes. We hope that a further prospective study will reveal the answer to this intriguing question.

This work was supported in part by a grant from the MRC.

\section{References}

' Mertin J. Immunological aspects of multiple sclerosis. In: Delmotte P, Gonsette RE, eds. Immunological and Clinical Aspects of Multiple Sclerosis. Lancaster: MTP Press, 1984:29-38.

${ }^{2}$ Weiner HL, Hauser SL. Neuroimmunology I: Immunoregulation in neurological disease. Ann Neurol 1982;11:437-49.

${ }^{3}$ Fishman RA. Cerebrospinal Fluid in Diseases of the Nervous System. Philadelphia: WB Saunders, 1980.

${ }^{4}$ Thompson EJ. Laboratory diagnosis of multiple sclerosis: immunological and biochemical aspects. $\mathrm{Br}$ Med Bull 1977;33:28-33.

5 Thompson EJ, Kaufmann $\mathbf{P}$, Shortman RC, Rudge $\mathbf{P}$ McDonald WI. Oligoclonal immunoglobulins and plasma cells in spinal fluid of patients with multiples sclerosis. Br Med J 1979;i:16-17.

${ }^{6}$ McDonald WI, Halliday AM. Diagnosis and classification of multiple sclerosis. Br Med Bull 197T 33:4-9.

${ }^{7}$ Kurtzke JF. Further notes on disability evaluation in multiple sclerosis, with scale modifications Neurol(Minn) 1965;15:654-61.

${ }^{8}$ Schumacher GA, Beebe G, Kibler RF, et al. Ann $N \dot{Y}$ Acad Sci 1965;122:552-68.

${ }^{9}$ Bollengier F, Lowenthal A, Henrotin W. Bound and free light chains in subacute sclerosing panencephalitis and multiple sclerosis serum and cerebrospinal fluid. $Z$ Klin Chem Klin Biochem 1975;13:305-10.

${ }^{10}$ Bollengier $F$. Bound and free light chains in serum from patients affected with various neurological diseases. $J$ Clin Chem Clin Biochem 1979;17:45-49.

" Kolar OJ, Rice PH, Jones FH, Defalque RJ, Kincaid J. Cerebrospinal fluid immunoelectrophoresis in multiple sclerosis. J Neurol Sci 1980;47:221-30. 